\title{
Fixed point theorems in locally convex spaces and a nonlinear integral equation of mixed type
}

Fuli Wang* and Hua Zhou

"Correspondence:

win-fully@163.com

School of Mathematics and Physics,

Changzhou University, Changzhou,

Jiangsu 213164, China

\section{Springer}

\begin{abstract}
In this paper, we provide a new approach for discussing the solvability of a class of operator equations by establishing fixed point theorems in locally convex spaces. Our results are obtained extend some Krasnosel'skii type fixed point theorems. As an application, we investigate the existence and global attractivity of solutions for a general nonlinear integral equation of mixed type of Urysohn and Volterra.
\end{abstract}

MSC: 47H10; 45G10; 45D05

Keywords: fixed point theorem; locally convex space; nonlinear integral equation

\section{Introduction}

As an example of algebraic settings, the captivating Krasnosel'skii's fixed point theorem (see [1] or [2], p.31) leads to the consideration of fixed points for the sum of two operators. It asserts that, if $M$ is a bounded, closed, and convex subset of a Banach space $X$ and $A, B$ are two mappings from $M$ into $X$ such that $A$ is compact and $B$ is a contraction, $A(M)+B(M) \subseteq M$, then $A+B$ has at least one fixed point in $M$. Since then, there has been a vast literature dealing with the improvements of such a result. For instance, in locally convex spaces or in Fréchet spaces all kinds of extensions and generalizations of Krasnosel'skii's fixed point theorem have been obtained by some authors (see e.g. [3-11] etc.).

As a more general consideration, there is another setting which leads one to investigate the fixed points for so-called nonautonomous type superposition operators, that is, to study the existence of solutions to the following operator equation:

$$
x=F(x, A x),
$$

for given operators $A: X \rightarrow Y$ and $F: X \times Y \rightarrow X$, where $X$ and $Y$ are given spaces. In the framework of Banach spaces, the solvability of equation (1.1) has been considered by one of the authors of this paper in $[12,13]$.

In the present paper, we will establish some new fixed point theorems for equation (1.1) in the framework of locally convex spaces. Our results extend some Krasnosel'skii type

(C) 2015 Wang and Zhou. This article is distributed under the terms of the Creative Commons Attribution 4.0 International License (http://creativecommons.org/licenses/by/4.0/), which permits unrestricted use, distribution, and reproduction in any medium, provided you give appropriate credit to the original author(s) and the source, provide a link to the Creative Commons license, and indicate if changes were made. 
fixed point theorems in the previous literature. As an application we will also study, respectively, the existence and the global attractivity of solutions for the following quite general nonlinear integral equation:

$$
x(t)=f\left(t, x(t), \int_{0}^{\infty} u(t, s, x(s)) d s, \int_{0}^{t} v(t, s, x(s)) d s\right), \quad t \geq 0,
$$

where $x$ belongs to $C\left(\mathbb{R}_{+}, \mathbb{R}^{d}\right)$, the space of all continuous functions defined on $\mathbb{R}_{+}:=[0, \infty)$ with values in the real Euclid space $\mathbb{R}^{d}$ of $d$ dimensions. Equation (1.2) is called a mixed type nonlinear integral equation since within its form an operator of Urysohn type and an operator of Volterra type appear.

Our results may also be applied to find the existence and uniqueness of solutions of differential equations of fractional order with integral boundary conditions [14].

Definition 1.1 (see [15], Definition 1) The solutions of equation (1.2) are said to be globally attractive, if for arbitrary solutions $x_{1}(t)$ and $x_{2}(t)$ of equation (1.2) it follows that $\lim _{t \rightarrow \infty}\left|x_{1}(t)-x_{2}(t)\right|=0$.

It should be noted that by means of a Krasnosel'skii type fixed point theorem, in the space $C\left(\mathbb{R}_{+}, \mathbb{R}^{d}\right)$ Avramescu and Vladimirescu [16] discussed the following nonlinear integral equation of mixed type:

$$
x(t)=q(t)+\int_{0}^{t} v(t, s, x(s)) d s+\int_{0}^{\infty} u(t, s, x(s)) d s .
$$

On the one hand, in the Banach space $\mathrm{BC}\left(\mathbb{R}_{+}\right)$consisting of all real functions continuous and bounded on $\mathbb{R}_{+}$, by means of the measure of noncompactness Hu and Yan [15], and Olszowy [17], respectively, discussed the following two equations:

$$
x(t)=f\left(t, x(t), \int_{0}^{t} v(t, s, x(s)) d s\right), \quad x(t)=f\left(t, x(t), \int_{0}^{\infty} u(t, s, x(s)) d s\right) .
$$

On the other hand, owing to the deficiency of a single measure of noncompactness in the space $C\left(\mathbb{R}_{+}, \mathbb{R}^{d}\right)$, the technique of a family of measures of noncompactness in conjunction with the Schauder-Tychonoff's fixed point theorem was applied by Olszowy [11, 18].

Since the calculating of measures of noncompactness is very hard in the majority of locally convex spaces, in the present paper we will show that by establishing some new fixed point results the use of the technique of measures of noncompactness may be avoided. Our goals in this paper are to establish new fixed point theorems for the solvability of equation (1.1) in locally convex spaces, and to study under what conditions equation (1.2) is solvable in $C\left(\mathbb{R}_{+}, \mathbb{R}^{d}\right)$ by applying our new theorems, and under what conditions the solutions of equation (1.2) are globally attractive. The results obtained will provide a new approach for discussing a class of operator equations in locally convex spaces.

The organization of this paper is as follows. In Section 2, we establish the fixed point theorems for equation (1.1) in locally convex spaces. In Section 3, we prove the existence and the global attractivity of solutions for equation (1.2). 


\section{Fixed point theorems in locally convex spaces}

Throughout this section, $X$ will denote a Hausdorff locally convex topological vector space, and $\left\{|\cdot|_{\rho}\right\}_{\rho \in \Lambda}$ a family of seminorms which generates the topology of $X$.

Let $\mathcal{U}$ be the neighborhood system of the origin obtained from $\Lambda$. Thus if $U \in \mathcal{U}$, there is a finite number of seminorms $\rho_{1}, \rho_{2}, \ldots, \rho_{n}$ in $\Lambda$ and real numbers $r_{1}, r_{2}, \ldots, r_{n}$ such that $U=\bigcap_{i=1}^{n} r_{i} V\left(\rho_{i}\right)$, where $V(\rho)=\left\{x:|x|_{\rho}<1\right\}$.

A mapping $T: X \rightarrow X$ is said to be a $|\cdot|_{\rho}$-contraction for $\rho \in \Lambda$ if there exists $\alpha_{\rho} \in[0,1)$ such that $\left|T x_{1}-T x_{2}\right|_{\rho} \leq \alpha_{\rho}\left|x_{1}-x_{2}\right|_{\rho}$ for all $x_{1}, x_{2} \in X$.

Theorem 2.1 Let $X$ be sequentially complete, and let $Y$ be a topological space. Suppose that $M$ is a closed and convex subset of $X$, and the operators $A: M \rightarrow Y$ and $F: X \times Y \rightarrow X$ satisfy the following conditions:

(i) $A$ is continuous, and $A(M)$ is a relatively compact subset of $Y$;

(ii) $F$ is continuous, and for each $\rho \in \Lambda$ there exists $\alpha_{\rho} \in[0,1)$ such that

$$
\left|F\left(x_{1}, y\right)-F\left(x_{2}, y\right)\right|_{\rho} \leq \alpha_{\rho}\left|x_{1}-x_{2}\right|_{\rho}
$$

for all $x_{1}, x_{2} \in X$ and $y \in Y$;

(iii) $z=F(z, A x), x \in M \Rightarrow z \in M$.

Then there is a point $x$ in $M$ such that $F(x, A x)=x$.

Proof For a given $y \in A(M)$ by assumption (ii) the mapping $F(\cdot, y)$ defined by $z \mapsto F(z, y)$ is a $|\cdot|_{\rho}$-contraction for each $\rho \in \Lambda$, so it has a unique fixed point in $X$ according to [3], Theorem 2.2. Now, let us denote by $J: A(M) \rightarrow X$ the mapping which assigns each $y \in A(M)$ to the unique point in $X$ such that $J y=F(J y, y)$. Accordingly, the mapping $J$ is well defined.

For a given $\rho \in \Lambda$, and arbitrary $y, y_{0}$ in $A(M)$, we have the estimate

$$
\begin{aligned}
\left|J y-J y_{0}\right|_{\rho} & =\left|F(J y, y)-F\left(J y_{0}, y_{0}\right)\right|_{\rho} \\
& \leq\left|F(J y, y)-F\left(J y_{0}, y\right)\right|_{\rho}+\left|F\left(J y_{0}, y\right)-F\left(J y_{0}, y_{0}\right)\right|_{\rho} \\
& \leq \alpha_{\rho}\left|J y-J y_{0}\right|_{\rho}+\left|F\left(J y_{0}, y\right)-F\left(J y_{0}, y_{0}\right)\right|_{\rho},
\end{aligned}
$$

it follows that

$$
\left|J y-J y_{0}\right|_{\rho} \leq\left(1-\alpha_{\rho}\right)^{-1}\left|F\left(J y_{0}, y\right)-F\left(J y_{0}, y_{0}\right)\right|_{\rho} .
$$

Let $U=\bigcap_{i=1}^{n} r_{i} V\left(\rho_{i}\right)$ be a given neighborhood of the origin in $X$. From the continuity of $F$ it is inferred that there exists a neighborhood $W_{y_{0}}$ of $y_{0} \in Y$ such that, for all $y \in W_{y_{0}} \cap A(M)$,

$$
\left|F\left(J y_{0}, y\right)-F\left(J y_{0}, y_{0}\right)\right|_{\rho_{i}}<1-\alpha_{\rho_{i}} \quad(i=1, \ldots, n)
$$

which implies that

$$
\left|J y-J y_{0}\right|_{\rho_{i}} \leq\left(1-\alpha_{\rho_{i}}\right)^{-1}\left|F\left(J y_{0}, y\right)-F\left(J y_{0}, y_{0}\right)\right|_{\rho_{i}}<1
$$


Thus, we obtain

$$
W_{y_{0}} \cap A(M) \subset J^{-1}\left(J y_{0}+U\right),
$$

and therefore $J$ is continuous.

By assumption (iii), for every $x \in M$ we infer that $z=J A x \in M$ owing to $z=F(z, A x)$, i.e. $J A(M) \subset M$. Since $A$ and $J$ are all continuous, the composite operator $J A$ is continuous on $M$; and $J A(M)$ is relatively compact by assumption (i). Now applying the SchauderTychonoff fixed point theorem (refer to [3], Theorem 2.1(b), or [19], p.96), we conclude that $J A$ has a fixed point $x \in M$ such that $J A x=x$, which implies that

$$
F(x, A x)=F(J A x, A x)=J A x=x .
$$

This completes the proof.

The following corollary shows that our above result extends the Krasnosel'skii fixed point theorem in locally convex spaces.

Corollary 2.2 Let $X$ be sequentially complete, and let $M$ be a closed and convex subset of $X$. Suppose that the operators $A: M \rightarrow X$ and $B: X \rightarrow X$ satisfy the following conditions:

(i) $A$ is continuous, and $A(M)$ is relatively compact;

(ii) $B$ is $a|\cdot|_{\rho}$-contraction for each $\rho \in \Lambda$;

(iii) $y=B y+A x, x \in M \Rightarrow y \in M$.

Then there is a point $x$ in $M$ such that $A x+B x=x$.

Since the assumption (iii) of Theorem 2.1 sometimes is hard to verify in actual applications, we next establish a Schaefer type fixed point theorem for equation (1.2).

Theorem 2.3 Let $X$ be sequentially complete, and let $Y$ denote a topological space. Suppose that the operators $A: X \rightarrow Y$ and $F: X \times Y \rightarrow X$ are continuous and satisfy the following conditions:

(i) A maps bounded sets of $X$ into relatively compact ones of $Y$;

(ii) for each $\rho \in \Lambda$ there exists $\alpha_{\rho} \in[0,1)$ such that

$$
\left|F\left(x_{1}, y\right)-F\left(x_{2}, y\right)\right|_{\rho} \leq \alpha_{\rho}\left|x_{1}-x_{2}\right|_{\rho}
$$

for all $x_{1}, x_{2} \in X$ and $y \in A(X)$.

Then, either

(a) there is a point $x$ in $X$ such that $x=F(x, A x)$, or

(b) the set $\{x \in X: x=\lambda F(x / \lambda, A x)\}$ is unbounded for some $\lambda \in(0,1)$.

Proof As in the proof of Theorem 2.1, let us denote by $J: A(X) \rightarrow X$ the mapping which assigns each $y \in A(X)$ to the unique point in $X$ such that $J y=F(J y, y)$. We know that $J$ is well defined and continuous.

Since the composite operator $J A: X \rightarrow X$ is continuous and maps bounded sets into relatively compact ones, by Schaefer's theorem [20] we find that (a) either the equation 
$x=\lambda J A x$ has a solution for $\lambda=1$, that is,

$$
x=J A x=F(J A x, A x)=F(x, A x) ;
$$

or (b) the set of all such solutions

$$
\{x \in X: x=\lambda J A x\}=\left\{x \in X: \frac{x}{\lambda}=F\left(\frac{x}{\lambda}, A x\right)\right\},
$$

is unbounded for some $\lambda \in(0,1)$. This completes the proof.

Corollary 2.4 Let $X$ be sequentially complete. Suppose that the two operators $A, B: X \rightarrow X$ satisfy the following conditions:

(i) $A$ is continuous and maps bounded sets into relatively weakly compact ones;

(ii) $B$ is $a|\cdot|_{\rho}$-contraction for each $\rho \in \Lambda$.

Then, either

(a) there is a point $x$ in $X$ such that $x=A x+B x$, or

(b) the set $\{x \in X: x=\lambda A x+\lambda B(x / \lambda)\}$ is unbounded for some $\lambda \in(0,1)$.

\section{The existence and global attractivity of solutions for a nonlinear integral equation of mixed type}

Throughout this section, we assume that the real Euclid space of $d$ dimensions $\mathbb{R}^{d}$ is endowed with an arbitrary norm $|\cdot|$, and the space $C\left(\mathbb{R}_{+}, \mathbb{R}^{d}\right)$ is endowed with the family of seminorms

$$
|x|_{T}:=\sup _{t \in[0, T]}|x(t)|, \quad T>0 .
$$

It is well known that $C\left(\mathbb{R}_{+}, \mathbb{R}^{d}\right)$ is a locally convex space and become a Fréchet space furnished with the distance

$$
d(x, y)=\sum_{k=1}^{\infty} \frac{1}{2^{k}} \frac{|x-y|_{k}}{1+|x-y|_{k}}
$$

Remark 3.1 Let us recall the following facts which will be crucial in our next considerations:

(a) a nonempty subset $M$ of $C\left(\mathbb{R}_{+}, \mathbb{R}^{d}\right)$ is said to be bounded if for each $T>0$ there exists a $L_{T}>0$ such that $|x|_{T} \leq L_{T}$ for all $x \in M$;

(b) the set $\left\{x \in C\left(\mathbb{R}_{+}, \mathbb{R}^{d}\right):|x(t)| \leq h(t)\right\}$ is a nonempty, bounded, closed, and convex subset of $C\left(\mathbb{R}_{+}, \mathbb{R}^{d}\right)$, where $h: \mathbb{R}_{+} \rightarrow \mathbb{R}_{+}$is a continuous function;

(c) a sequence $\left(x_{n}\right)_{n \in \mathbb{N}}$ is convergent to $x$ in $C\left(\mathbb{R}_{+}, \mathbb{R}^{d}\right)$ if and only if $\left|x_{n}-x\right|_{T} \rightarrow 0$ for each $T>0$, i.e. $\left(x_{n}\right)_{n \in \mathbb{N}}$ is uniformly convergent to $x$ on the interval $[0, T]$;

(d) a bounded subset $M$ of $C\left(\mathbb{R}_{+}, \mathbb{R}^{d}\right)$ is relatively compact if and only if, for each $T>0$, the restrictions to $[0, T]$ of all functions from $M$ form an equicontinuous set.

We will, respectively, prove the existence of solutions and the global attractivity of solutions for equation (1.2) under the following assumptions: 
(H1) $u: \mathbb{R}_{+}^{2} \times \mathbb{R}^{d} \rightarrow \mathbb{R}^{d}$ is continuous, and there exists a continuous function $\kappa_{1}: \mathbb{R}_{+}^{2} \rightarrow \mathbb{R}_{+}$ such that, for all $(t, s) \in \mathbb{R}_{+}^{2}$ and $x \in \mathbb{R}^{d}$,

$$
|u(t, s, x)| \leq \kappa_{1}(t, s)|x|
$$

$(\mathcal{H} 2)$ there exists a continuous function $h: \mathbb{R}_{+} \rightarrow \mathbb{R}_{+}$such that the improper integral $\int_{0}^{\infty} \kappa_{1}(t, s) h(s) d s$ is uniformly convergent on all parameters $t \in[0, T]$ for each $T>0$, i.e. for arbitrarily given $\varepsilon>0$ there exists $S=S(\varepsilon, T)>0$ such that, for all $t \in[0, T]$,

$$
\int_{S}^{\infty} \kappa_{1}(t, s) h(s) d s<\varepsilon
$$

(H3) $v: \Delta \times \mathbb{R}^{d} \rightarrow \mathbb{R}^{d}$ is continuous, and there exists a continuous function $\kappa_{2}: \Delta \rightarrow \mathbb{R}_{+}$ such that

$$
|v(t, s, x)| \leq \kappa_{2}(t, s)|x|
$$

for all $(t, s) \in \Delta$ and $x \in \mathbb{R}^{d}$, where $\Delta:=\left\{(t, s) \in \mathbb{R}_{+}^{2}: 0 \leq t \leq s\right\} ;$

(H4) $f: \mathbb{R}_{+} \times\left(\mathbb{R}^{d}\right)^{3} \rightarrow \mathbb{R}^{d}$ is continuous, and there exist three continuous functions $\alpha$ : $\mathbb{R}_{+} \rightarrow[0,1), \beta: \mathbb{R}_{+} \rightarrow \mathbb{R}_{+}$and $\gamma: \mathbb{R}_{+} \rightarrow \mathbb{R}_{+}$such that

$$
\left|f\left(t, x_{1}, \bar{u}_{1}, \bar{v}_{1}\right)-f\left(t, x_{2}, \bar{u}_{2}, \bar{v}_{2}\right)\right| \leq \alpha(t)\left|x_{1}-x_{2}\right|+\beta(t)\left|\bar{u}_{1}-\bar{u}_{2}\right|+\gamma(t)\left|\bar{v}_{1}-\bar{v}_{2}\right|,
$$

for all $x_{i}, \bar{u}_{i}, \bar{v}_{i} \in \mathbb{R}^{d}(i=1,2)$ and $t \in \mathbb{R}_{+}$;

$(\mathcal{H} 5)$ the following inequality holds for all $t \in \mathbb{R}^{+}$:

$$
|f(t, 0,0,0)|+\alpha(t) h(t)+\beta(t) \int_{0}^{\infty} \kappa_{1}(t, s) h(s) d s+\gamma(t) \int_{0}^{t} \kappa_{2}(t, s) h(s) d s \leq h(t)
$$

$(\mathcal{H} 6)$ the following conditions are satisfied:

$$
\lim _{t \rightarrow \infty} \frac{\beta(t)}{1-\alpha(t)} \int_{0}^{\infty} \kappa_{1}(t, s) h(s) d s=0 \quad \text { and } \quad \lim _{t \rightarrow \infty} \frac{\gamma(t)}{1-\alpha(t)} \int_{0}^{t} \kappa_{2}(t, s) h(s) d s=0
$$

Under the above assumptions, we define a nonempty, bounded, closed, and convex subset of $C\left(\mathbb{R}_{+}, \mathbb{R}^{d}\right)$ as follows:

$$
M:=\left\{x \in C\left(\mathbb{R}_{+}, \mathbb{R}^{d}\right):|x(t)| \leq h(t)\right\},
$$

and set $H_{T}:=\sup _{t \in[0, T]} h(t)$. We will apply Theorem 2.1 to prove the existence of solutions to equation (1.2). To this end, we introduce the following two lemmas.

Lemma 3.2 If $(\mathcal{H} 1)$ and $(\mathcal{H} 2)$ are satisfied, then the operator $U$ defined by

$$
(U x)(t):=\int_{0}^{\infty} u(t, s, x(s)) d s
$$

is continuous on $M$ and $U(M)$ is relatively compact in $C\left(\mathbb{R}_{+}, \mathbb{R}^{d}\right)$. 
Proof For all $x \in M$, from $(\mathcal{H} 1)$ and $(\mathcal{H} 2)$ we obtain

$$
\left|\int_{0}^{\infty} u(t, s, x(s)) d s\right| \leq \int_{0}^{\infty}|u(t, s, x(s))| d s \leq \int_{0}^{\infty} \kappa_{1}(t, s) h(s) d s .
$$

Since for a given $x \in M$ and for each $t \geq 0$ the function $u(t, \cdot, x(\cdot))$ is integrable on $\mathbb{R}_{+}$, and $u$ is continuous, the function $U x$ is continuous on $\mathbb{R}^{+}$. Accordingly, the operator $U$ : $C\left(\mathbb{R}_{+}, \mathbb{R}^{d}\right) \rightarrow C\left(\mathbb{R}_{+}, \mathbb{R}^{d}\right)$ is well defined.

Let $T>0$ be fixed. By setting $K_{1}:=\max \left\{\kappa_{1}(t, s):(t, s) \in[0, T] \times[0, T]\right\}$, from $(\mathcal{H} 1)$ we obtain, for all $x \in M$ and $t \in[0, T]$,

$$
\begin{aligned}
|(U x)(t)| & \leq \int_{0}^{\infty}|u(t, s, x(s))| d s \leq \int_{0}^{T} \kappa_{1}(t, s)|x(s)| d s+\int_{T}^{\infty} \kappa_{2}(t, s)|x(s)| d s \\
& \leq \int_{0}^{T} \kappa_{1}(t, s) h(s) d s+\int_{T}^{\infty} \kappa_{1}(t, s) h(s) d s \\
& \leq T K_{1} H_{T}+\sup _{t \in[0, T]} \int_{0}^{\infty} \kappa_{1}(t, s) h(s) d s,
\end{aligned}
$$

which shows that the restrictions to $[0, T]$ of all functions from $U(M)$ form an uniformly bounded set.

For $\forall \varepsilon>0$, by $(\mathcal{H} 2)$ there exists $S=S(\varepsilon, T)>0$ such that

$$
\sup _{t \in[0, T]} \int_{S}^{\infty} \kappa_{1}(t, s) h(s) d s \leq \frac{\varepsilon}{3} .
$$

By the uniform continuity of $u$ on the set

$$
\Pi_{T}:=\left\{(t, s, x) \in \mathbb{R}_{+}^{2} \times \mathbb{R}^{d}: t \in[0, T], s \in[0, S],|x| \leq H_{T}\right\}
$$

there exists $\delta=\delta(\varepsilon)>0$ such that

$$
\left|u\left(t_{1}, s_{1}, x_{1}\right)-u\left(t_{2}, s_{2}, x_{2}\right)\right| \leq \frac{\varepsilon}{3 S}
$$

for $\left(t_{i}, s_{i}, x_{i}\right) \in \Pi_{T}(i=1,2)$ with $\left|\left(t_{1}, s_{1}, x_{1}\right)-\left(t_{2}, s_{2}, x_{2}\right)\right| \leq \delta$. By combining (3.2) and (3.3) it follows that

$$
\begin{aligned}
\left|(U x)\left(t_{1}\right)-(U x)\left(t_{2}\right)\right| \leq & \int_{0}^{\infty}\left|u\left(t_{1}, s, x(s)\right)-u\left(t_{2}, s, x(s)\right)\right| d s \\
\leq & \int_{0}^{s}\left|u\left(t_{1}, s, x(s)\right)-u\left(t_{2}, s, x(s)\right)\right| d s \\
& +\int_{S}^{\infty}\left(\left|u\left(t_{1}, s, x(s)\right)\right|+\left|u\left(t_{2}, s, x(s)\right)\right|\right) d s \\
\leq & \frac{\varepsilon}{3}+2 \sup _{t \in[0, T]} \int_{S}^{\infty} \kappa_{1}(t, s) h(s) d s \leq \varepsilon,
\end{aligned}
$$

for $t_{1}, t_{2} \in[0, T]$ with $\left|t_{1}-t_{2}\right| \leq \delta$ and all $x \in M$. This shows the restrictions to $[0, T]$ of all functions from $U(M)$ form an equicontinuous set, and the arbitrariness of $T>0$ implies that the set $U(M)$ is relatively compact. 
Let $\left(x_{n}\right)_{n \in \mathbb{N}} \subseteq M$ be a sequence with $x_{n} \rightarrow x$. The uniform continuity of $u$ on $\Pi_{T}$ and the convergence of $\left(x_{n}\right)_{n \in \mathbb{N}}$ implies that there exists $n_{0}=n_{0}(\varepsilon, T)$ such that, for all $n \geq n_{0}$ and $(t, s) \in[0, T] \times[0, S]$

$$
\left|u\left(t, s, x_{n}(s)\right)-u(t, s, x(s))\right| \leq \frac{\varepsilon}{3 S} .
$$

Thus, from (3.2) and (3.4) we obtain

$$
\begin{aligned}
& \left|\left(U x_{n}\right)(t)-(U x)(t)\right| \\
& \quad \leq \int_{0}^{S}\left|u\left(t, s, x_{n}(s)\right)-u(t, s, x(s))\right| d s+2 \sup _{t \in[0, T]} \int_{S}^{\infty} \kappa_{1}(t, s) h(s) d s \\
& \quad \leq \varepsilon
\end{aligned}
$$

which implies that $\left|U x_{n}-U x\right|_{T} \rightarrow 0$, and the arbitrariness of $T>0$ implies the continuity of $U$ is proved.

Lemma 3.3 If $(\mathcal{H} 3)$ is satisfied, then the operator $V$ defined by

$$
(V x)(t):=\int_{0}^{t} v(t, s, x(s)) d s
$$

is continuous on $M$ and $V(M)$ is relatively compact in $C\left(\mathbb{R}_{+}, \mathbb{R}^{d}\right)$.

Proof Let $\Delta_{T}=\{(t, s): 0 \leq s \leq t \leq T\}$ for an arbitrarily given $T>0$. It is easily known that the function $V x$ is continuous on $\mathbb{R}^{+}$for a given $x \in M$ since $v$ is continuous and the function $v(t, \cdot x(\cdot))$ is integrable on $[0, t]$ for each $t \in[0, T]$. Thus the operator $V$ : $C\left(\mathbb{R}_{+}, \mathbb{R}^{d}\right) \rightarrow C\left(\mathbb{R}_{+}, \mathbb{R}^{d}\right)$ is well defined.

By setting $K_{2}:=\max \left\{\kappa_{2}(t, s):(t, s) \in \Delta_{T}\right\}$, from (H3) we obtain, for all $x \in M$ and $t \in$ $[0, T]$,

$$
\begin{aligned}
|(V x)(t)| & \leq \int_{0}^{t}|v(t, s, x(s))| d s \leq \int_{0}^{T} \kappa_{2}(t, s)|x(s)| d s \\
& \leq \int_{0}^{T} \kappa_{2}(t, s) h(s) d s \leq T K_{2} H_{T},
\end{aligned}
$$

which shows that the restrictions to $[0, T]$ of all functions from $V(M)$ form an uniformly bounded set.

By the uniform continuity of $v$ on the set

$$
\Omega_{T}:=\left\{(t, s, x) \in \Delta \times \mathbb{R}^{d}:(t, s) \in \Delta_{T},|x| \leq H_{T}\right\},
$$

for $\forall \varepsilon>0$ there exists $\delta=\delta(\varepsilon, T)>0$ such that

$$
\left|v\left(t_{1}, s_{1}, x_{1}\right)-v\left(t_{2}, s_{2}, x_{2}\right)\right| \leq \frac{\varepsilon}{T+K_{2} H_{T}}
$$


for all $\left(t_{i}, s_{i}, x_{i}\right) \in \Omega_{T}(i=1,2)$ with $\left|\left(t_{1}, s_{1}, x_{1}\right)-\left(t_{2}, s_{2}, x_{2}\right)\right| \leq \delta$. Let us choose $\delta \leq$ $\varepsilon /\left(T+K_{2} H_{T}\right)$. It follows that, for each $x \in M$ and $\left|t_{1}-t_{2}\right| \leq \delta$ with $t_{1}<t_{2}$,

$$
\begin{aligned}
\left|(V x)\left(t_{1}\right)-(V x)\left(t_{2}\right)\right| & \leq \int_{0}^{t_{1}}\left|v\left(t_{1}, s, x(s)\right)-v\left(t_{2}, s, x(s)\right)\right| d s+\int_{t_{1}}^{t_{2}}\left|v\left(t_{2}, s, x(s)\right)\right| d s \\
& \leq \frac{T \varepsilon}{T+K_{2} H_{T}}+\int_{t_{1}}^{t_{2}} \kappa_{2}(t, s) h(s) d s \\
& \leq \frac{T \varepsilon}{T+K_{2} H_{T}}+K_{2} H_{T} \delta \leq \varepsilon,
\end{aligned}
$$

which shows that the restrictions to $[0, T]$ of all functions from $V(M)$ form an equicontinuous set, and the arbitrariness of $T>0$ implies that the set $V(M)$ is relatively compact.

Let $\left(x_{n}\right)_{n \in \mathbb{N}}$ be a sequence in $M$ with $x_{n} \rightarrow x \in M$. Since $v$ is uniformly continuous on $\Omega_{T}$, it follows that for $\forall \varepsilon>0$ there exists $n_{0}=n_{0}(\varepsilon, T)$ such that, for all $n \geq n_{0}$ and $(t, s) \in \Delta_{T}$,

$$
\left|v\left(t, s, x_{n}(s)\right)-v(t, s, x(s))\right| \leq \frac{\varepsilon}{T} .
$$

Thus, for $\forall t \in[0, T]$ we have

$$
\begin{aligned}
\left|\left(V x_{n}\right)(t)-(V x)(t)\right| & \leq \int_{0}^{t}\left|v\left(t, s, x_{n}(s)\right)-v(t, s, x(s))\right| d s \\
& \leq \int_{0}^{T}\left|v\left(t, s, x_{n}(s)\right)-v(t, s, x(s))\right| d s \leq \varepsilon,
\end{aligned}
$$

which implies that $\left|V x_{n}-V x\right|_{T} \rightarrow 0$, and the arbitrariness of $T>0$ implies the continuity of $V$ is proved.

We are now in a position by applying our Theorem 2.1 to prove the existence and global attractivity of solutions of equation (1.2).

Theorem 3.4 Under the assumptions $(\mathcal{H} 1)-(\mathcal{H} 5)$ equation $(1.2)$ has at least one solution $x=x(t)$ which belongs to the spaces $C\left(\mathbb{R}_{+} ; \mathbb{R}^{d}\right)$; furthermore if $(\mathcal{H} 6)$ is also satisfied then the solutions of equation (1.2) are globally attractive.

Proof Let us set $X:=C\left(\mathbb{R}_{+}, \mathbb{R}^{d}\right)$ and $Y:=C\left(\mathbb{R}_{+}, \mathbb{R}^{d}\right) \times C\left(\mathbb{R}_{+}, \mathbb{R}^{d}\right)$. Let $M$ be defined by (3.1). The operators $A: M \rightarrow Y$ and $F: X \times Y \rightarrow X$ are, respectively, defined by

$$
(A x)(t):=((U x)(t),(V x)(t)), \quad F(x, y)(t):=f(t, x(t), \bar{u}(t), \bar{v}(t)), \quad t \in \mathbb{R}_{+},
$$

for all $x \in X$ and $y=(\bar{u}, \bar{v}) \in Y$ with $\bar{u}, \bar{v} \in X$. Our proving is divided into several steps: in (1)-(3) we prove the existence; in (4) we prove the global attractivity.

(1) According to Lemma 3.2 and Lemma 3.3, for an arbitrarily given $x \in X$ we easily infer $A x=(U x, V x) \in Y$ since $(U x)(t)$ and $(V x)(t)$ are all continuous functions defined on $\mathbb{R}_{+}$; and the continuity of operator $A$ is also easily obtained from the continuity of operators $U$ and $V$.

Further, from Lemma 3.2 and Lemma 3.3 we infer that $A(M) \subseteq U(M) \times V(M)$ is relatively compact by the Tychonoff product theorem, and therefore the condition (i) of Theorem 2.1 is satisfied. 
(2) Obviously, for arbitrarily given $x \in X$ and $y \in Y$ the continuity of $F(x, y)(t)$ on $\mathbb{R}_{+}$may easily be inferred from continuity of the function $f(t, x(t), \bar{u}(t), \bar{v}(t))$.

Let $x_{0} \in X$ and $y_{0}=\left(\bar{u}_{0}, \bar{v}_{0}\right) \in Y$. Suppose that $\left(x_{n}\right)_{n \in \mathbb{N}}$ is contained in $X$ and $\left(y_{n}\right)_{n \in \mathbb{N}}$ is contained in $Y$, respectively, such that $x_{n} \rightarrow x_{0}$ and $y_{n} \rightarrow y_{0}$. By taking $y_{n}=\left(\bar{u}_{n}, \bar{v}_{n}\right)$ it follows that $\bar{u}_{n} \rightarrow \bar{u}_{0}$ and $\bar{v}_{n} \rightarrow \bar{v}_{0}$. Let us set

$$
\alpha_{T}:=\sup _{t \in[0, T]} \alpha(t), \quad \beta_{T}:=\sup _{t \in[0, T]} \beta(t), \quad \gamma_{T}:=\sup _{t \in[0, T]} \gamma(t) .
$$

For an arbitrarily given $T>0$, from $(\mathcal{H} 4)$ we infer that

$$
\begin{aligned}
& \left|F\left(x_{n}, y_{n}\right)-F\left(x_{0}, y_{0}\right)\right|_{T} \\
& \quad=\sup _{t \in[0, T]}\left|f\left(t, x_{n}(t), \bar{u}_{n}(t), \bar{v}_{n}(t)\right)-f\left(t, x_{0}(t), \bar{u}_{0}(t), \bar{v}_{0}(t)\right)\right| \\
& \quad \leq \alpha_{T}\left|x_{n}-x_{0}\right|_{T}+\beta_{T}\left|\bar{u}_{n}-u_{0}\right|_{T}+\gamma_{T}\left|\bar{v}_{n}-v_{0}\right|_{T},
\end{aligned}
$$

which implies the operator $F$ is continuous on $X \times Y$.

Further, for $\forall x_{1}, x_{2} \in X$ and $\forall y=(\bar{u}, \bar{v}) \in Y$ by $(\mathcal{H} 4)$ we obtain

$$
\begin{aligned}
\left|F\left(x_{1}, y\right)-F\left(x_{2}, y\right)\right|_{T} & =\sup _{t \in[0, T]}\left|f\left(t, x_{1}(t), \bar{u}(t), \bar{v}(t)\right)-f\left(t, x_{2}(t), \bar{u}(t), \bar{v}(t)\right)\right| \\
& \leq \alpha_{T}\left|x_{1}-x_{2}\right|_{T}
\end{aligned}
$$

for each $T>0$, which implies that the condition (ii) of Theorem 2.1 is satisfied.

(3) We are going to prove that if for $x \in M$ there is $z \in X$ such that

$$
z(t)=f(t, z(t),(U x)(t),(V x)(t))
$$

then $z \in M$. In fact, from $(\mathcal{H} 4)$ we have the following estimate:

$$
\begin{aligned}
|z(t)| & \leq|f(t, 0,0,0)|+|f(t, z(t),(U x)(t),(V x)(t))-f(t, 0,0,0)| \\
& \leq|f(t, 0,0,0)|+\alpha(t)|z(t)|+\beta(t)|(U x)(t)|+\gamma(t)|(V x)(t)|,
\end{aligned}
$$

and by $(\mathcal{H} 5)$ we have

$$
\begin{aligned}
|z(t)| & \leq \frac{1}{1-\alpha(t)}[|f(t, 0,0,0)|+\beta(t)|(U x)(t)|+\gamma(t)|(V x)(t)|] \\
& \leq \frac{1}{1-\alpha(t)}\left[|f(t, 0,0,0)|+\beta(t) \int_{0}^{\infty} \kappa_{1}(t, s) h(s) d s+\gamma(t) \int_{0}^{t} \kappa_{2}(t, s) h(s) d s\right] \\
& \leq h(t) .
\end{aligned}
$$

Accordingly, we obtain $z \in M$ and the condition (iii) is satisfied. Now applying Theorem 2.1 we conclude the proof of the existence part.

(4) Suppose that $x_{1}$ and $x_{2}$ are two solutions of equation (1.2), then by $(\mathcal{H} 4)$ we have

$$
\begin{aligned}
& \left|x_{1}(t)-x_{2}(t)\right| \\
& \quad \leq \alpha(t)\left|x_{1}(t)-x_{2}(t)\right|+\beta(t)\left|\left(U x_{1}\right)(t)-\left(U x_{2}\right)(t)\right|+\gamma(t)\left|\left(V x_{1}\right)(t)-\left(V x_{2}\right)(t)\right|,
\end{aligned}
$$


which implies that

$$
\begin{aligned}
& \left|x_{1}(t)-x_{2}(t)\right| \\
& \quad \leq \frac{\beta(t)}{1-\alpha(t)}\left(\left|\left(U x_{1}\right)(t)\right|+\left|\left(U x_{2}\right)(t)\right|\right)+\frac{\gamma(t)}{1-\alpha(t)}\left(\left|\left(V x_{1}\right)(t)\right|+\left|\left(V x_{2}\right)(t)\right|\right) \\
& \quad \leq \frac{2 \beta(t)}{1-\alpha(t)} \int_{0}^{\infty} \kappa_{1}(t, s) h(s) d s+\frac{2 \gamma(t)}{1-\alpha(t)} \int_{0}^{t} \kappa_{2}(t, s) h(s) d s .
\end{aligned}
$$

Thus, by $(\mathcal{H} 6)$ we obtain

$$
\lim _{t \rightarrow \infty}\left|x_{1}(t)-x_{2}(t)\right|=0
$$

By now we showed that the solution of equation (1.2) is globally attractive and finish the proof.

\section{Competing interests}

The authors declare that they have no competing interests.

\section{Authors' contributions}

All authors contributed equally and significantly in writing the article. All authors read and approved the final manuscript.

\section{Acknowledgements}

This research was supported by Natural Science Foundation of the Jiangsu Higher Education Institutions of China (No. 15KJB110001).

Received: 21 May 2015 Accepted: 30 November 2015 Published online: 14 December 2015

\section{References}

1. Krasnosel'skii, MA: Two remarks on the method of successive approximations. Usp. Mat. Nauk 10, 123-127 (1955) (in Russian)

2. Smart, DR: Fixed Point Theorems. Cambridge University Press, Cambridge (1980)

3. Cain, GL Jr., Nashed, MZ: Fixed points and stability for a sum of two operators in locally convex spaces. Pac. J. Math. 39(3), 581-592 (1971)

4. Sehgal, VM, Singh, SP: On a fixed point theorem of Krasnoselskii for locally convex spaces. Pac. J. Math. 62(2), 561-567 (1976)

5. Hoa, LH, Schmitt, K: Fixed point theorems of Krasnosel'skii type in locally convex spaces and applications to integral equations. Results Math. 25, 290-314 (1994)

6. Avramescu, C: Some remarks on a fixed point theorem of Krasnoselskii. Electron. J. Qual. Theory Differ. Equ. 2003, 5 (2003)

7. Barroso, CS, Teixeira, EV: A topological and geometric approach to fixed points results for sum of operators and applications. Nonlinear Anal. 60, 625-650 (2005)

8. Ngoc, LTP, Long, NT: On a fixed point theorem of Krasnosel'skii type and application to integral equations. Fixed Point Theory Appl. 2006, 30847 (2006)

9. Vladimirescu, C: Remark on Krasnoselskii's fixed point theorem. Nonlinear Anal. 71, 876-880 (2009)

10. Ben Amar, A, Cherif, MA, Mnif, M: Fixed-point theory on a Frechet topological vector space. Int. J. Math. Math. Sci. 2011, $390720(2011)$

11. Olszowy, L: Fixed point theorems in the Fréchet space $C\left(\mathbb{R}_{+}\right)$and functional integral equations on an unbounded interval. Appl. Math. Comput. 218, 9066-9074 (2012)

12. Wang, F: A fixed point theorem for nonautonomous type superposition operators and integrable solutions of a general nonlinear functional integral equation. J. Inequal. Appl. 2014, 487 (2014)

13. Wang, F: Solvability of a general nonlinear integral equation in $L^{1}$-spaces by means of a weak measure of noncompactness. J. Integral Equ. Appl. 27(2), 273-287 (2015)

14. Nanware, JA, Dhaigude, DB: Existence and uniqueness of solutions of differential equations of fractional order with integral boundary conditions. J. Nonlinear Sci. Appl. 7, 246-254 (2014)

15. Hu, X, Yan, J: The global attractivity and asymptotic stability of solution of a nonlinear integral equation. J. Math. Anal. Appl. 321, 147-156 (2006)

16. Avramescu, C, Vladimirescu, C: An existence result of asymptotically stable solutions for an integral equation of mixed type. Electron. J. Qual. Theory Differ. Equ. 2005, 25 (2005)

17. Olszowy, L: On solutions of functional-integral equations of Urysohn type on an unbounded interval. Math. Comput. Model. 47, 1125-1133 (2008)

18. Olszowy, L: Existence of mild solutions for the semilinear nonlocal problem in Banach spaces. Nonlinear Anal. 81, 211-223 (2013)

19. Agarwal, RP, Meehan, M, O’Regan, D: Fixed Point Theory and Applications. Cambridge University Press, Cambridge (2001)

20. Schaefer, H: Über die Methode der a priori-Schranken. Math. Ann. 129, 415-416 (1955) 